\title{
Volumen plaquetario medio, índice de neutrófilos/linfocitos e índice de trombocitos/linfocitos para determinar el diagnóstico y/o pronóstico en niños con mordedura de serpiente \\ Mean platelet volume, neutrophil to lymphocyte ratio and platelet to lymphocyte ratio in determining the diagnosis or outcome in children with snakebite
}

Prof. Asist. Fesih Aktar y y Prof. Asist. Recep Tekin ${ }^{b}$

\section{RESUMEN}

Objetivo. Evaluar la relación entre las variables de volumen plaquetario medio (VPM), índice de neutrófilos/linfocitos (INL) e índice de trombocitos/linfocitos (ITL) y el diagnóstico o la predicción del desenlace en los niños con intoxicación por mordedura de serpiente.

Métodos. Se realizó una evaluación retrospectiva de niños con diagnóstico de intoxicación por mordedura de serpiente y un grupo de referencia de sujetos sanos. Se clasificó a los pacientes en tres grupos de intoxicación: leve, moderada y grave.

Resultados. Se incluyeron 142 niñosen el estudio. La leucocitosis $(p=0,003)$, la neutrofilia $(p=0,026)$ y la trombocitopenia $(p=0,034)$ fueron significativamente más frecuentes en los casos de intoxicación por mordedura de serpiente grave; sin embargo, no se hallaron diferencias estadísticamente significativas en relación con el VPM, el INL y el ITL entre los diferentes grupos de intoxicación por mordedura de serpiente. La media del VPM, el INL y el ITL era significativamente mayor entre los niños con mordedura de serpiente en comparación con los controles sanos. Conclusiones. Según nuestros resultados, el uso del VPM, el INL y el ITL podría servir para el diagnóstico como marcadores inflamatorios en los casos de intoxicación por mordedura de serpiente.

Palabras clave: volumen plaquetario medio; indice de neutrófilos/ linfocitos; índice de trombocitos/linfocitos; intoxicación por mordedura de serpiente; niño.

http:/ / dx.doi.org/10.5546/ aap.2017.576

Texto completo en inglés:

http:/ / dx.doi.org/10.5546/ aap.2017.eng.576

Cómo citar: Aktar F, Tekin R. Volumen plaquetario medio, índice de neutrófilos/linfocitos e índice de trombocitos/linfocitos para determinar el diagnóstico y/o pronóstico en niños con mordedura de serpiente. Arch Argent Pediatr 2017;115(6):576-580.

a. Facultad de Medicina de Dicle University, Departamento de Enfermedades Infecciosas Pediátricas, Diyarbakir, Turquía.

b. Facultad de Medicina de Dicle University, Departamento de Enfermedades Infecciosas y Microbiología Clínica, Diyarbakir, Turquía.

Correspondencia: Prof. Asist. Fesih Aktar: fesihaktar@yahoo.com

Financiamiento: Ninguno.

Conflicto de intereses: Ninguno que declarar.

Recibido: 13-10-2016

Aceptado: 7-6-2017

\section{INTRODUCCIÓN}

En la práctica clínica, no se dispone de marcadores diagnósticos de mordedura de serpiente confiables..$^{1-3}$ Por lo tanto, el diagnóstico preciso de intoxicación por mordedura de serpiente requiere la identificación precisa de la mordedura de serpiente y el estudio de los datos clínicos relativos a la intoxicación por mordedura de ofidio venenoso. ${ }^{3}$ Los datos de laboratorio de la intoxicación por mordedura de ofidio venenoso se obtienen a partir de los graves efectos del veneno a nivel citotóxico, inflamatorio y del sistema hematopoyético. ${ }^{4,5} \mathrm{El}$ veneno de serpiente agrava los efectos sistémicos, entre otros, la inflamación y la proliferación de leucocitos en la zona de la mordedura. Según informes recientes, existe una asociación entre la inflamación y el aumento de parámetros de laboratorio novedosos, tales como el volumen plaquetario medio (VPM), el índice de neutrófilos / linfocitos (INL), el índice de trombocitos/linfocitos (ITL) y el recuento de trombocitos. ${ }^{4-6}$

En ningún estudio previo se han evaluado el VPM, el INL y el ITL en pacientes con mordedura de serpiente. Nuestra hipótesis fue que la intoxicación por mordedura de serpiente, que es una afección inflamatoria, podría generar un aumento de los marcadores sistémicos de inflamación, tales como el VPM, el INL y el ITL. El objetivo de este estudio fue evaluar la relación entre el VPM, el INL y el ITL y el diagnóstico o la predicción del desenlace entre los niños con intoxicación por mordedura de serpiente.

\section{MATERIALES Y MÉTODOS Pacientes}

Este estudio retrospectivo se llevó a cabo en los consultorios pediátricos; y se incluyó a niños menores de 18 años. Se obtuvieron las historias clínicas de todos los pacientes con antecedentes de mordedura de serpiente entre junio de 2006 y septiembre de 2014 según los registros del 
hospital. Se realizó el diagnóstico de mordedura de serpiente si los sujetos habían visto al ofidio o si el aspecto de la zona de la herida punzante era claramente indicativo de mordedura de serpiente.

Se obtuvieron los datos de los pacientes a partir de las fichas de seguimiento completadas al momento de la hospitalización y de los registros del hospital. Se recolectó información acerca de la edad, el sexo, la gravedad de la intoxicación por mordedura de ofidio venenoso y los datos de laboratorio.

\section{Recolección de datos y comparaciones}

Se calcularon el INL y el ITL como el cociente de neutrófilos:linfocitos y de trombocitos:linfocitos, respectivamente. Los sujetos sanos eran niños que asistieron al hospital para controles de rutina. Se excluyó del grupo de referencia a los niños con signos de infección, enfermedad sistémica o inflamación.

En el estudio se incluyó a los pacientes con mordedura de serpiente y a un grupo de referencia de niños sanos de edad y sexo correspondiente al grupo de pacientes. Se compararon la concentración de leucocitos y hemoglobina, el recuento de neutrófilos, linfocitos y trombocitos, y el VPM, el INL y el ITL entre ambos grupos.

\section{Definiciones}

La gravedad de la intoxicación por mordedura de ofidio venenoso se determinó según los criterios adoptados por Otero. ${ }^{7}$ Intoxicación por mordedura de ofidio venenoso leve se define como tumefacción de uno o dos segmentos de la extremidad mordida y un aumento inferior a 4 $\mathrm{cm}$ de la circunferencia de la extremidad en la zona de la mordedura, sin necrosis ni síntomas sistémicos. Intoxicación por mordedura de ofidio venenoso moderada se define como tumefacción de dos o tres segmentos de la extremidad mordida y un aumento superior a $4 \mathrm{~cm}$ de la circunferencia de la extremidad y hemorragia local en la zona de la mordedura, sin necrosis local ni síntomas sistémicos o con síntomas mínimos. Intoxicación por mordedura de ofidio venenoso grave se define como tumefacción más allá de la extremidad mordida (hacia el tronco), ampollas, hemorragia local, necrosis o síndrome compartimental y signos clínicos sistémicos, entre otros, hemorragia sistémica múltiple, hipotensión o choque, coagulación intravascular

TABLA 1. Características demográficas y datos de laboratorio de los grupos por gravedad de la mordedura de serpiente

\begin{tabular}{|c|c|c|c|c|}
\hline & Leve & Moderada & Grave & \\
\hline & $\begin{array}{c}\text { Media + DE o } \\
\text { mediana (intervalo) } \\
\text { o cantidad (\%) } \\
n=28\end{array}$ & $\begin{array}{c}\text { Media + DE o } \\
\text { mediana (intervalo) } \\
\text { o cantidad (\%) } \\
n=70\end{array}$ & $\begin{array}{c}\text { Media + DE o } \\
\text { mediana (intervalo) } \\
\text { o cantidad (\%) } \\
n=44\end{array}$ & Valor $p$ \\
\hline \multicolumn{5}{|l|}{ Características demográficas } \\
\hline Edad (meses) & $120(36-181)$ & $108(25-170)$ & $121(35-181)$ & 0,325 \\
\hline \multirow[t]{2}{*}{ Sexo } & $9(32,1)$ & $21(30)$ & $16(36,4)$ & 0,763 \\
\hline & $19(67,9)$ & $49(70)$ & $28(63,6)$ & \\
\hline \multicolumn{5}{|c|}{ Datos de laboratorio al momento de la hospitalización } \\
\hline $\mathrm{L}(\mathrm{K} / \mu \mathrm{L})$ & $10,9 \pm 4,8$ & $15,2 \pm 6,6$ & $16,5 \pm 7,9$ & 0,003 \\
\hline Neutrófilos $\left(10^{3} / \mu 1\right)$ & $8,8 \pm 5,2$ & $12,6 \pm 6,8$ & $13,2 \pm 6,9$ & 0,026 \\
\hline Linfocitos $\left(10^{3} / \mu 1\right)$ & $2,1 \pm 1,2$ & $1,9 \pm 1,2$ & $2,4 \pm 1,7$ & 0,527 \\
\hline Hemoglobina (g/dl) & $12,7 \pm 1,1$ & $13,3 \pm 1,5$ & $13,0 \pm 2,5$ & 0,191 \\
\hline Recuento de trombocitos $\left(10^{3} / \mu 1\right)$ & $304,2 \pm 85,1$ & $300,9 \pm 99,8$ & $257,3 \pm 99,9$ & 0,034 \\
\hline $\mathrm{VPM}(\mathrm{fl})$ & $8,14 \pm 1,35$ & $8,59 \pm 1,58$ & $8,41 \pm 1,78$ & 0,303 \\
\hline INI & $7,64 \pm 8,97$ & $9,94 \pm 8,86$ & $8,52 \pm 6,38$ & 0,196 \\
\hline ITI & $221,1 \pm 215,0$ & $216,0 \pm 144,4$ & $164,1 \pm 122,1$ & 0,141 \\
\hline
\end{tabular}

L: leucocitos, VPM: volumen plaquetario medio, INL: índice de neutrófilos/linfocitos, ITL: índice de trombocitos/linfocitos,

DE: desviación estándar 
diseminada, insuficiencia renal, hemorragia cerebral o insuficiencia sistémica múltiple.

\section{Criterios de exclusión}

Se excluyó a los pacientes con trastornos crónicos, anemia u otras enfermedades hematológicas e infecciones bacterianas agudas. También se excluyó del estudio a los pacientes que no vieron a la serpiente y no presentaron marcas de mordedura de serpiente o que presentaron otras formas de mordeduras desconocidas que no coincidían con mordedura de serpiente.

El Comité de Ética de la Facultad de Medicina de la Universidad de Dicle aprobó el protocolo de investigación.

\section{Análisis estadístico}

Todos los análisis de datos se realizaron con el paquete estadístico para ciencias sociales (Statistical Package for Social Sciences, SPSS), versión 18.0, para Windows. Se utilizó la prueba de Kolmogorov-Smirnov para evaluar la normalidad de la distribución de los datos continuos. Las variables numéricas con distribución normal se describieron como media más/menos desviación estándar. Las variables numéricas con distribución normal se compararon con la prueba t de Student. Se utilizó la prueba $\chi^{2}$ para comparar las variables categóricas entre los grupos. Se empleó el análisis de la varianza (ANOVA) unidireccional o la prueba de KruskalWallis para comparar los tres subgrupos. La incidencia acumulada se calculó para todo el período del estudio, junto con los intervalos de confianza del 95\%. Los valores de $p<0,05$ se consideraron estadísticamente significativos.

\section{RESULTADOS}

Se realizó una evaluación retrospectiva de 142 niños con diagnóstico de intoxicación por mordedura de serpiente y 142 sujetos sanos. La media de la edad de los pacientes fue de $110,1 \pm 37,5$ meses; $96 / 142(67,6 \%)$ de los pacientes eran varones. La media de la edad del grupo de referencia fue de 97,8 \pm 44,3 meses; 95/142 (66,9\%) de los sujetos eran varones. No se observaron diferencias significativas en la media de la edad ni en la distribución por sexo en el grupo de referencia $(p>0,05)$.

Se observó intoxicación por mordedura de serpiente leve en $28(19,8 \%)$ pacientes; moderada, en $70(49,2 \%)$; y grave, en $44(31 \%)$. La leucocitosis $(p=0,003)$, la neutrofilia $(p=0,026)$ y la trombocitopenia $(p=0,034)$ fueron significativamente más frecuentes en el grupo de intoxicación por mordedura de serpiente grave; sin embargo, no se hallaron diferencias estadísticamente significativas en relación con el VPM, el INL y el ITL $(p>0,05)$ (Tabla 1$)$.

En el grupo de pacientes se observaron valores de VPM, INL, ITL, leucocitos, neutrófilos y linfocitos significativamente más elevados y recuento de trombocitos más bajos al momento de la hospitalización en comparación con el grupo de referencia $(p<0,05)($ Tabla 2$)$.

\section{DISCUSIÓN}

En un estudio anterior nuestro, se observó que la duración de la hospitalización, la ocurrencia en un entorno rural, el recuento de leucocitos, el índice de AST-ALT, la creatina quinasa, la hipoproteinemia, la hipoalbuminemia y la hipocalcemia están asociados con la gravedad

TABLA 2. Datos de laboratorio estudiados y grupos de referencia

\begin{tabular}{|c|c|c|c|}
\hline \multirow{3}{*}{$\begin{array}{l}\text { Variables } \\
\text { hematológicas }\end{array}$} & Grupo del estudio & Grupo de referencia & \multirow[b]{3}{*}{ Valor $p$} \\
\hline & (n: 142) & (n: 142) & \\
\hline & $\begin{array}{c}\text { Media + DE o } \\
\text { mediana del intervalo }\end{array}$ & $\begin{array}{c}\text { Media + DE o } \\
\text { mediana del intervalo }\end{array}$ & \\
\hline $\mathbf{L}(\mathrm{K} / \mu \mathrm{l})$ & $14,9 \pm 7,05$ & $8,59 \pm 2,15$ & $<0,001$ \\
\hline Recuento de trombocitos $\left(10^{3} / \mu \mathrm{l}\right)$ & $287 \pm 99,3$ & $330 \pm 87,6$ & $<0,001$ \\
\hline Neutrófilos $\left(10^{3} / \mu 1\right)$ & $11,3(5,96-16,4)$ & $4,14(3,28-5,54)$ & $<0,001$ \\
\hline Linfocitos $\left(10^{3} / \mu 1\right)$ & $1,85(1,15-2,74)$ & $2,74(2,32-3,51)$ & $<0,001$ \\
\hline VPM (fl) & $8,11(7,48-9,05)$ & $7,63(7,19-8,39)$ & 0,002 \\
\hline INL & $5,98(2,52-13,0)$ & $1,43(1,09-1,90)$ & $<0,001$ \\
\hline ITI & $146,5(105,1-259,4)$ & $113,3(55,0-136,6)$ & $<0,001$ \\
\hline
\end{tabular}

L: leucocitos, VPM: volumen plaquetario medio, INL: índice de neutrófilos/linfocitos, ITL: índice de trombocitos/linfocitos, DE: desviación estándar 
de la mordedura de serpiente. El recuento de leucocitos y el índice de AST-ALT pueden usarse como criterios de seguimiento de los niños con mordedura de serpiente. ${ }^{1}$ Según el estudio actual, el VPM, el INL y el ITL podrían servir para el diagnóstico como marcadores inflamatorios de la mordedura de serpiente. Hasta donde sabemos, este es el primer estudio que investigó la relación entre la mordedura de serpiente y los parámetros séricos del hemograma completo en niños.

La estructura del veneno es compleja e incluye muchas toxinas y proteínas que causan efectos inflamatorios sistémicos, necrosantes y hemorrágicos y que podrían representar varias de las manifestaciones inflamatorias observadas en la mordedura de serpiente. . $-6,8^{2}$ El VPM, el INL y el ITL se miden como parte de los análisis de sangre de rutina en muchos laboratorios sin que esto implique costos adicionales. ${ }^{4,9}$ El VPM se asocia con la activación y la función de los trombocitos en las afecciones inflamatorias. ${ }^{6,9}$ Los trombocitos son componentes vitales de la hemostasia normal y liberan varias citocinas proinflamatorias. ${ }^{4,6,9}$ El VPM puede aumentar cuando se produce inflamación leve y puede disminuir en los casos de inflamación más grave debido al consumo demandante de trombocitos en las zonas de inflamación. ${ }^{4}$ El VPM refleja las afecciones proinflamatorias, en las que las citocinas proinflamatorias regulan el proceso de inflamación. ${ }^{4}$ Las mordeduras de serpiente generan actividad inflamatoria considerable, tanto a nivel local como sistémico. En este estudio, se observó que el VPM era significativamente más alto en los pacientes con mordedura de serpiente que en los sujetos sanos. Por lo tanto, se planteó la hipótesis de que el VPM podría servir para el diagnóstico de la mordedura de serpiente.

El VPM actúa como una proteína de la fase aguda en algunas afecciones inflamatorias, según la gravedad de la inflamación sistémica. ${ }^{4,9}$ En este estudio, también se compararon los valores del VPM y la gravedad de la enfermedad y no se halló ninguna relación entre estas variables en los niños con mordedura de serpiente.

El INL es una marcador de la inflamación sistémica. ${ }^{9,10}$ Aumenta en las afecciones inflamatorias pero no se ha estudiado específicamente en los pacientes con mordedura de serpiente. El INL sirve para el diagnóstico de la inflamación. 4,6,9-11 Se halló una relación significativa entre el INL y la intoxicación por mordedura de serpiente en comparación con los sujetos sanos del grupo de referencia. Por lo tanto, podría usarse el
INL como indicador del marcador inflamatorio de mordedura de serpiente.

También podría emplearse para predecir la mortalidad y establecer un pronóstico. El INL es un factor adecuado para predecir la gravedad de la enfermedad en algunas afecciones. ${ }^{9-11}$ Aún no se ha elucidado el mecanismo subyacente de la asociación entre el INL y la gravedad de la enfermedad, el cual sigue siendo controversial. El recuento elevado de neutrófilos tal vez dependa de la fiebre activada por la IL-1 $\beta$, la neutrofilia y la migración de neutrófilos hacia los tejidos. ${ }^{12}$ En este estudio, no se observó una relación entre el INL y la gravedad de la enfermedad en los pacientes con mordedura de serpiente.

La intoxicación por mordedura de serpiente se asocia con trombosis, hemostasia e inflamación. ${ }^{13} \mathrm{El}$ ITL es otro indicador de la respuesta inflamatoria sistémica que se obtiene a partir de los análisis de sangre de rutina y puede usarse para evaluar el proceso inflamatorio en la mordedura de serpiente. La cantidad de trombocitos puede aumentar en condiciones de inflamación y hemorragia, lo que puede producir un aumento de la expresión de las citocinas proinflamatorias y trombocitosis. ${ }^{6,11-13}$ El recuento elevado de trombocitos puede ser un reflejo de la inflamación de fondo, y la reducción del recuento de linfocitos puede representar señales de inflamación no controlada. Por lo tanto, el aumento del ITL puede servir como marcador de la inflamación sistémica. ${ }^{6,10}$ En algunos estudios se informó que el aumento del recuento de trombocitos y la reducción del recuento de linfocitos están asociados con tumores malignos, arteriopatía coronaria y diversas enfermedades inflamatorias. ${ }^{9,14,15}$ Feng y col. informaron que el ITL estaba asociado con una sobrevida menor en los casos de cáncer esofágico. ${ }^{14}$ Acmaz y col. demostraron que el ITL estaba elevado en los pacientes con cáncer de endometrio en comparación con los sujetos del grupo de referencia e informaron que este marcador inflamatorio también puede usarse para el diagnóstico. ${ }^{15}$ En el estudio actual, el ITL era significativamente más alto en los pacientes con mordedura de serpiente que en los sujetos sanos del grupo de referencia. Se ha informado que el ITL está asociado con la gravedad de la enfermedad, la sobrevida, el diagnóstico diferencial, el pronóstico y el seguimiento en varios estudios sobre diversos estados de enfermedad. ${ }^{9,13-15}$ En este estudio no se observó una relación entre el ITL y la gravedad de la enfermedad.

Este estudio presenta ciertas limitaciones 
potenciales. Este fue un estudio retrospectivo con una pequeña cantidad de casos y se llevó a cabo en una sola institución; además, no se estudiaron los subtipos de linfocitos ni otros indicadores del sistema inflamatorio, como las citocinas, la proteína C-reactiva y la sedimentación, y los antecedentes cardíacos de los pacientes no presentaban particularidades.

\section{CONCLUSIONES}

Los niveles de VPM, INL e ITL pueden ser útiles para el diagnóstico de mordedura de serpiente, el cual a veces se torna difícil para los pediatras. En este estudio no se observó una relación entre el VPM, el INL y el ITL y la gravedad de la mordedura de serpiente. Por lo tanto, se concluye que el VPM, el INL y el ITL no serían indicadores pronósticos confiables en los niños con mordedura de serpiente. Sin embargo, se requieren investigaciones futuras con mayor cantidad de pacientes para establecer si existe una relación entre estos biomarcadores y la gravedad de la forma clínica en los niños con mordedura de serpiente.

\section{REFERENCIAS}

1. Aktar F, Aktar S, Yolbas I, et al. Evaluation of risk factors and follow-up criteria for severity of snakebite in children. Iran J Pediatr 2016;26(4):e5212.

2. Chippaux JP. Epidemiology of snakebites in Europe: a systematic review of the literature. Toxicon 2012;59(1): 86-99.

3. Gold BS, Dart RC, Barish RA. Bites of venomous snakes. N Engl J Med 2002;347(5):347-56.

4. Santhosh MS, Sundaram MS, Sunitha K, et al. Viper venom- induced oxidative stress and activation of inflammatory cytokines: a therapeutic approach for over looked issues of snakebite management. Inflamm Res 2013;62(7):721-31.

5. Sunitha K, Hemshekhar M, Thushara RM, et al. Inflammation and oxidative stress in viper bite: an insight within and beyond. Toxicon 2015;98:89-97.

6. Mannaioni PF, Di Bello MG, Masini E. Platelets and inflammation: role of platelet-derived growth factor, adhesion molecules and histamine. Inflamm Res 1997;4 6(1):4-18.

7. Otero-Patiño R. Epidemiological, clinical and therapeutic aspects of Bothrops asper bites. Toxicon 2009;54(7): 9981011.

8. Tekin R, Sula B, Cakır G, et al. Comparison of snakebite cases in children and adults. Eur Rev Med Pharmacol Sci 2015;19(14):2711-6.

9. Kemal Y, Yucel I, Ekiz K, et al. Elevated serum neutrophile to lymphocyte and platelet to lymphocyte ratios could be useful in lung cancer diagnosis. Asian Pac J Cancer Prev 2014;15(6):2651-4.

10. Bhat T, Teli S, Rijal J, et al. Neutrophil to lymphocyte ratio and cardiovascular diseases: a review. Expert Rev Cardiovasc Ther 2013;11(1):55-9.

11. Ulu S, Ulu MS, Bucak A, et al. Neutrophil-to-lymphocyte ratio as a new, quick, and reliable indicator for predicting diagnosis and prognosis of idiopathic sudden sensorineural hearing loss. Otol Neurotol 2013;34(8):1400-4.

12. Dinarello CA. Biologic basis for interleukin-1 in disease. Blood 1996;87(6):2095-147.

13. McCleary RJ, Kini RM. Snake bites and hemostasis/ thrombosis. Thromb Res 2013;132(6):642-6.

14. Feng JF, Huang Y, Chen QX. Preoperative platelet lymphocyte ratio (PLR) is superior to neutrophil lymphocyte ratio (NLR) as a predictive factor in patients with esophageal squamous cell carcinoma. World J Surg Oncol 2014;12:58.

15. Acmaz G, Aksoy H, Unal D, et al. Are neutrophil/ lymphocyte and platelet/lymphocyte ratios associated with endometrial precancerous and cancerous lesions in patients with abnormal uterine bleeding? Asian Pac J Cancer Prev 2014;15(4):1689-92.

\title{
Participación de la mujer en la autoría de revistas pediátricas latinoamericanas
}

\section{Female authorship in Latin American pediatric journals}

\author{
Dra. Paula Otero ${ }^{a, b}$, Cecilia Marcos ${ }^{b}$ y Dr. Fernando Ferrero ${ }^{b, c}$
}

\author{
a. Hospital Italiano de Buenos Aires. \\ b. Archivos Argentinos de Pediatría. \\ c. Hospital General de Niños Pedro de Elizalde.
}

Correspondencia: Dr. Fernando Ferrero,fferrero@intramed.net.

Financiamiento: Ninguno.

Conflicto de intereses: Ninguno que declarar.

Recibido: $19-1-2017$

Aceptado: 12-6-2017

\section{RESUMEN}

Introducción. La participación de la mujer en las ciencias se incrementa día a día. Se estimó la participación de la mujer en las 3 publicaciones pediátricas latinoamericanas indexadas en PubMed.

Métodos. Se identificaron todos los artículos de Archivos Argentinos de Pediatría, Jornal de Pediatria y Revista Chilena de Pediatría correspondientes al año 2015 y se determinó el primer y último autor, y el número total de autores por sexo.

Resultados. Se identificaron 329 artículos. De los 1432 autores, $59,9 \%$ eran mujeres. El $54,4 \%$ de los primeros autores y el $48 \%$ de los últimos autores eran mujeres. No hubo diferencia significativa en la proporción de mujeres autoras entre las 